\title{
Varroose : mise en évidence de la résistance du parasite aux acaricides par la méthode de «détermination du temps létal moyen»
}

\author{
JP Faucon, P Drajnudel, C Fléché \\ Laboratoire de pathologie des petits ruminants et des abeilles, Cneva, Les Templiers, \\ 105, route des Chappes, BP 111, 06902 Sophia-Antipolis, France
}

(Reçu le 5 octobre 1995 ; accepté le 13 février 1996)

\begin{abstract}
Résumé - Depuis l'apparition de la varroose en France, différents acaricides ont été utilisés : l'amitraz, le fluvalinate, l'acrinathrine. Une méthode d'évaluation des résistances par comparaison des temps létaux a été mise au point. L'acaricide a été apporté soit avec un morceau calibré de médicament (Apistan ${ }^{\circledR}$, Apivar ${ }^{\circledR}$ ), soit en imprégnant un rectangle calibré de papier Whatmann $n^{\circ} 3$ d'une quantité connue. Dans une boîte transparente, cinq abeilles ont été mises en présence de l'acaricide durant 2 heures. Passé ce délai, cinq varroas ont été déposés sur les abeilles. Le temps pour que les parasites se décrochent de leur hôte a été déterminé. Selon les souches de varroa, le temps létal a varié de 6 à 1218 minutes pour le fluvalinate. Pour l'acrinathrine, une différence significative a été enregistrée en fonction de la résistance des varroas au fluvalinate. En ce qui concerne l'amitraz, aucune différence n'a été relevée.
\end{abstract}

Apis mellifera / varroose / acaricide / résistance / temps létal

\section{INTRODUCTION}

La varroose due à l'acarien Varroa jacobsoni Oud a fait son apparition il y a plus de 13 ans en France (Colin, 1983). Cette acariose a été principalement traitée par l'amitraz (Anti-varroa Schering ${ }^{\circledR}$ ) (Faucon et Fléché, 1988) et le fluvalinate (Apistan ${ }^{\circledR}$ ) (Borneck et Merle, 1990) depuis sa mise sur le marché en 1989. Des essais expérimentaux ont été effectués au rucher du Cneva de Sophia-Antipolis avec l'acrinathrine sous sa forme phytosanitaire Rufast ${ }^{\circledR}$
(Faucon, en cours de publication) dans le but de s'orienter vers de nouvelles molécules de traitement. Une autre spécialité à base d'amitraz (Apivar ${ }^{\circledR}$ ) (Richez et al, 1995) vient de recevoir une autorisation de mise sur le marché. L'utilisation fréquente de ces molécules, leur mode d'utilisation à libération lente, le non respect des consignes de traitements par les apiculteurs ont fait craindre très tôt l'apparition de résistances (Bassand, 1993). Celle-ci a été démontrée vis-à-vis du fluvalinate dans plusieurs pays européens (Lodesani et al, 1995 ; Fluri, 1995 ; 
Milani, 1995) dont la France, au printemps 1995 (Faucon et al, 1995). Cependant les taux d'infestation élevés annoncés périodiquement ne sont pas toujours le fait d'une résistance. La possibilité d'une erreur de traitement, d'une recontamination au voisinage de rucher non traités ou d'essaims sauvages ne doit pas être oubliée (Nelson et Jay, 1989; Sakofski et al, 1990) avec pour conséquence d'imputer à tort l'échec du traitement à une résistance. Dans ces conditions, il est important de détenir une méthode simple d'évaluation de la résistance du parasite aux acaricides de façon à déterminer avec certitude la cause des taux d'infestation élevés constatés et pour suivre l'extension du phénomène.

La méthode mise au point, plus simple que celles décrites par différents auteurs (Abed et Ducos de Lahitte, 1993 ; Vandame et al, 1995) compare le temps létal d'une souche de référence du Cneva de SophiaAntipolis aux temps létaux de diverses souches en provenance de colonies ayant un taux de parasitisme élevé.

\section{MATÉRIELS ET MÉTHODES}

\section{Matériels}

Des boîtes de Pétri jetables d'un diamètre de $5 \mathrm{~cm}$ ont été utilisées comme cagette d'expérimentation. Le couvercle a été découpé et aménagé avec un grillage de couleur blanche de maille 0,5 $\mathrm{mm}$ de façon à assurer la respiration des abeilles, permettre leur nourrissement et introduire les parasites par une maille découpée plus large. La jointure parfaite du couvercle et de la boîte a été assurée au moyen d'une pâte collante (fig 1).

Le temps létal vis-à-vis de trois acaricides a été recherché : fluvalinate (Apistan ${ }^{\circledR}$ ), amitraz (Apivar ${ }^{\circledR}$ ), acrinathrine (Rufast ${ }^{\circledR}$ ). L'acaricide a été apporté de deux manières différentes en fonction de sa spécificité : i) pour les médicaments Apistan ${ }^{(*)}$ et Apivar ${ }^{(\beta)}$, il a été découpé des bandes de $0,5 \mathrm{~cm}$ de haut et de largeur égale à celle de la lanière de médicament, correspondant res- pectivement à un poids moyen de $0,18 \mathrm{~g}$ $(0,16-0,20)$ et $0,39 \mathrm{~g}(0,36-0,41)$; ii) pour l'acaricide liquide, il a été découpé des bandes de papier Wathmann $n^{\circ} 3$ de $0,5 \times 3 \mathrm{~cm}$ imprégnées de $0,05 \mathrm{~mL}$ de Rufast ${ }^{\circledR}$ pur qui ont ensuite été séchées $\mathbf{3 0}$ minutes à l'air libre. Suivant le cas, I'un ou l'autre de ces dispositifs a été collé au fond et au centre de la boîte.

Les varroas provenaient : i) d'une souche considérée comme référence conservée sans traitement au Cneva de Sophia-Antipolis depuis 2 ans ; ii) de colonies ayant eu un traitement normal à l'Apistan ${ }^{\circledR}$ depuis 2 ans et effectuant des transhumances dans des régions de grande concentration apicole (souche 1); iii) de colonies pour lesquelles les apiculteurs annonçaient l'échec des traitements à l'Apistan ${ }^{\circledR}$ (souches 2 et 3). Le prélèvement des varroas s'est fait dans du couvain operculé (ce qui permet leur transport et une bonne survie des parasites), au stade larve ou prénymphe pour ne travailler que sur des femelles fondatrices. Seules les femelles adultes ont été utilisées. Leur manipulation s'est faite au moyen d'un pinceau. Toute femelle endomma-

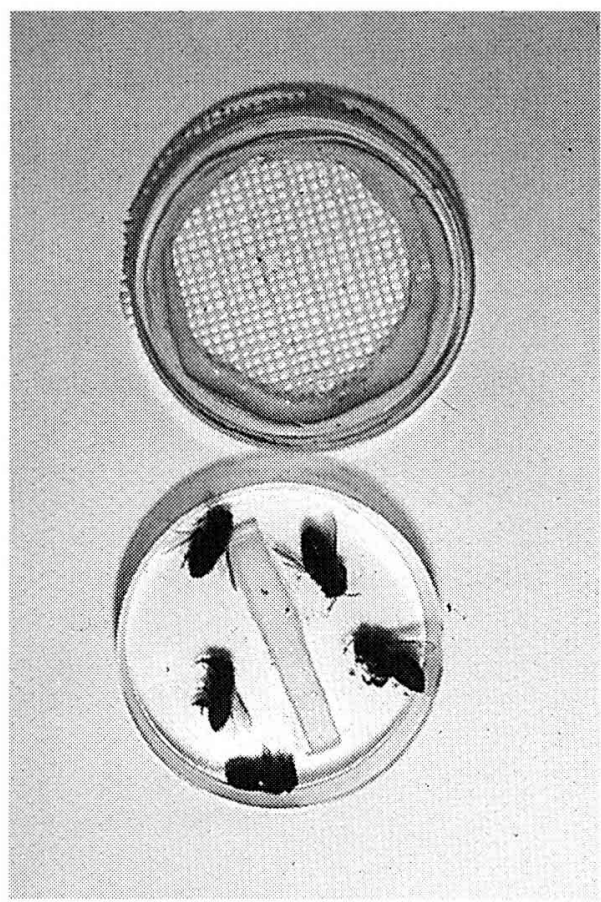

Fig 1. Uispositî́ expérimental pour la détermination du temps létal. 
gée ou présentant une faible vitalité a été éliminée. Avant leur utilisation pour le test, les femelles varroas ont été conservées dans une boîte de Pétri humidifiée par un coton et sur des nymphes d'abeilles (stade œil blanc).

Les abeilles utilisées pour les essais ont toutes été prélevées dans la même colonie non traitée depuis 6 mois. Elles étaient endormies au gaz carbonique pour permettre leur manipulation.

\section{Méthodes}

Cinq cagettes tests et cinq cagettes témoins ont été utilisées au minimum pour chaque essai conduit conformément au protocole suivant :

- temps $T_{0}$ : cinq abeilles endormies au gaz carbonique ont été diposées dans les cagettes en dehors du contact de l'acaricide ; les cagettes ont ensuite été mises à l'étuve $\left(35^{\circ} \mathrm{C}, 80 \%\right.$ d'hygrométrie) durant 2 heures pour permettre aux abeilles de se charger d'acaricide par contact ;

$-T+30$ minutes à $T+120$ minutes : nourrissement des abeilles avec du miel. Dans le même temps, les 50 varroas nécessaires pour l'essai ont été recherchés dans un rayon de couvain operculé et collectés dans la boîte de Pétri préparée à cet effet ;

$-T+120$, cinq parasites par cagette ont été apportés sur les abeilles. L'accrochage sur leur hôte a été vérifié.

Par la suite, le temps au bout duquel tous les parasites ont été retrouvés au fond de la cagette a été évalué par observation du fond de la boîte (toutes les 5 minutes durant la première heure, le plus souvent possible par la suite avec un maximum de 15 minutes). La comparaison entre les temps létaux de la souche de référence et les temps létaux des différentes souches testées (souches $1,2,3$ ) a été effectuée par le test $F$ pour la comparaison de plusieurs moyennes.

Les boîtes témoins ont été conduites de la même manière mais en l'absence de produit acaricide. Seul un morceau de papier Wathmann a été collé au fond de la boîte. Dans ce cas, la permanence de l'accrochage des parasites sur les abeilles a été vérifiée tout au long de l'essai.

Tableau I. Variation du temps létal moyen de trois souches $(1,2,3)^{a}$ de Varroa jacobsoni Oud, pour trois acaricides différents en comparaison avec une souche du Cneva de Sophia-Antipolis prise comme référence.

\begin{tabular}{|c|c|c|c|}
\hline $\begin{array}{l}\text { Souches } \\
\text { de varroa }\end{array}$ & $\begin{array}{c}\text { Nombre } \\
\text { de cagettes }\end{array}$ & $\begin{array}{l}\text { Temps létal } \\
\text { moyen (min) }\end{array}$ & $\begin{array}{c}\text { Valeurs } \\
\text { extrêmes (min) }\end{array}$ \\
\hline - & -- & & \\
\hline \multicolumn{4}{|l|}{ Apistan ${ }^{\otimes}$} \\
\hline Souche prise comme référence & 11 & $18,7 \pm 6,3$ & $9-28$ \\
\hline Souche 1 & 17 & $42,5 \pm 21,7$ & $6-78$ \\
\hline Souche 2 & 7 & $397,1 \pm 412,3$ & $33-1080$ \\
\hline Souche 3 & 13 & $544,7 \pm 312,8$ & $182-1218$ \\
\hline \multicolumn{4}{|l|}{ Rufast $^{\circledR}$} \\
\hline Souche de référence & 12 & $6,16 \pm 2,5$ & $3-11$ \\
\hline Souche 3 & 12 & $19,25 \pm 10,9$ & $6-78$ \\
\hline \multicolumn{4}{|l|}{ Apivar ${ }^{\circledR}$} \\
\hline Souche de référence & 10 & $26,1 \pm 8,5$ & $14-43$ \\
\hline \multirow[t]{2}{*}{ Souche 3 . } & 13 & $24 \pm 9,3$ & $13-47$ \\
\hline & & $\ldots$ & -.. \\
\hline
\end{tabular}




\section{RÉSULTATS}

L'évaluation des temps létaux moyens de la souche prise comme référence vis-à-vis des trois acaricides a été de 18,7 $( \pm 6,3)$ min pour le fluvalinate (Apistan $\left.{ }^{(B)}\right), 6,1( \pm 2,5)$ min pour l'acrinathrine (Rufast ${ }^{(\mathcal{B})}$ ) et 26,1 $( \pm 8,5)$ min pour l'amitraz (Apivar ${ }^{\circledR}$ ) (tableau I).

La comparaison de la souche prise comme référence vis-à-vis des souches 1 , 2, 3 a montré une différence significative pour l'Apistan ( $p=0,0001)$ et l'acrinathrine $(p=0,0006)$. Aucune différence significative $n$ 'a été mise en évidence entre la souche prise comme référence et la souche 3 pour l'Apivar ${ }^{\circledR}(p=0,59)$.

Pour les essais réalisés avec l'Apistan ${ }^{\circledR}$ sur des souches de varroas issues de colonies où dans certains cas, les traitements posaient des problèmes, le temps létal a varié de 6 à 1218 minutes, mettant en évidence un allongement significatif du temps létal moyen (tableau I).

Pour les essais effectués avec le Rufast $t^{(2)}$ et l'Apivar ${ }^{\circledR}$ sur la souche de varroas résistante au fluvalinate, le temps létal a varié respectivement de 6 à 78 minutes et de 13 à 47 minutes. Le temps létal moyen de la souche 3 par rapport à la souche prise comme référence a été modifié significativement pour le Rufast $\left.^{(}\right)$seulement (tableau I).

\section{DISCUSSION ET CONCLUSION}

La méthode mise au point a permis d'établir les temps létaux moyens vis-à-vis de trois acaricides utilisés dans la lutte contre la varroose, pour une souche de parasites considérée comme référence. En effet cette souche n'a subi aucun traitement depuis 2 ans. La colonie survit en maintenant un seuil d'infestation peu élevé (Peng et al, 1987), ce qui a rendu difficile la recherche des varroas dans le couvain et par conséquent la multiplication des essais.

À partir des temps létaux usuels établis, un suivi dans le temps et dans l'espace pourra être facilement mis en place afin de vérifier l'évolution de l'efficacité des traitements.

La différence du temps létal de la souche de référence et des temps létaux pour des parasites issus de colonies où dans certains cas les traitements étaient inefficaces (souches 1, 2 et 3 ) est significative. Elle confirme la résistance, en montrant par ailleurs des niveaux de résistance au fluvalinate différents selon les souches testées, et explique les difficultés de traitements rencontrées. Ces résultats sont à rapprocher de ceux enregistrés lors de l'enquête conduite dans la vallée de la Roya (région frontalière avec I'Italie) (Faucon, 1995), destinée à évaluer l'efficacité de l'Apistan ${ }^{\circledR}$. Les pertes d'efficacité avaient été retrouvées dans les ruchers exposés plein sud, donc là où les colonies entretenaient du couvain de façon plus intense. En raison de l'allongement du temps létal, le parasite conserve la possibilité de se laisser enfermer dans le couvain, ce qui augmente le nombre de cycles de multiplication.

L'acrinathrine est un acaricide appartenant à la famille des norpyrithrates, famille voisine de celle des pyréthrinoïdes à laquelle appartient le fluvalinate. Les traitements conduits au moyen de cette molécule ont montré leur efficacité en station expérimentale mais la comparaison des temps létaux entre la souche de référence et une souche déjà résistante au fluvalinate (souche 3) est significative et démontre un début de résistance à l'acrinathrine. Pour l'amitraz, aucune différence significative n'a été mise en évidence entre la souche de référence et une souche déjà résistante au fluvalinate.

Une enquête pour établir les temps létaux moyens de base des principaux acaricides utilisés et pour des parasites originaires de 
différentes régions sera conduite afin de posséder une référence ultérieure. Ainsi, cette méthode simple de mise en évidence de résistances de Varroa jacobsoni aux acaricides permettra le contrôle de l'efficacité des traitements pour une meilleure information des apiculteurs.

\section{REMERCIEMENTS}

Les auteurs remercient $M$ Pépin, pour ses conseils, et I Saint-Pierre, stagiaire de la faculté d'Orsay.

\section{Summary - Varroosis: demonstrating} resistance of parasites to acaricides by means of the 'determination of mean lethal time' method. A simple method of evaluation of Varroa jacobsoni resistance to acaricides (amitraz, fluvalinate and acrinathrin) was developed by comparison of lethal times with a reference strain from CNEVA Sophia Antipolis. This method reproduced the field conditions of the bee colony and kept the host-parasite relationship. The acaricide was administered either as a small piece of plastic strip (Apistan ${ }^{\circledR}$, Apivar $\left.{ }^{\circledR}\right)$, or by impregnation of a piece of Whatmann paper no 3 with a known quantity of acaricide. The strip was stuck on the bottom of a Petri dish covered with a net. Five bees were put in contact with the acaricide for 2 hours (fig 1 ). After that, five mites were introduced onto the bees. The time for the parasites to fall off their host was determined and compared to controls without acaricide. According to the different varroa strains, the lethal time varied from 6-1218 min with fluvalinate. The highest lethal times were registered from mites coming from apiaries where treatments with Apistan ${ }^{\circledR}$ were ineffective (table I). A significant difference was noted with acrinathrin, coincident with the degree of resistance of the mites to fluvalinate. With amitraz, no difference was found. This simple method, named 'deter- mination of average lethal time', can be applied to other acaricides and provide information on the development of resistance through time to beekeepers.

\section{Apis mellifera / varroa disease / acari- cides / resistance / lethal time}

Zusammenfassung - Varroose: Ermittlung von Resistenzen des Parasiten gegen Akarizide nach der Methode der "Bestimmung der mittleren Überlebensdauer". Als einfache Methode zur Bestimmung von Akarizidresistenz bei Varroa jacobsoni gegenüber Amitraz, Fluvalinat und Acrinathrin wurde die Überlebenszeit mit einer Referenzpopulation von CNEVA, Sophia Antipolis verglichen. Bei dieser Methode waren die Anwendungsbedingungen ähnlich wie im Bienenvolk und die WirtParasitbeziehung wurde aufrechterhalten. Das jeweilige Akarizid wurde entweder als kleiner Streifenabschnitt (Apistan, Apivar) oder durch Tränkung eines Papierstreifens (Whatmann, Nr 3) mit einer bekannten Akarizidmenge angewandt. Der Streifen wurde auf den Boden einer mit einem Netz verschlossenen Petrischale festgeheftet und darin fünf Arbeiterinnen zwei Stunden lang mit dem Akarizid in Berührung gebracht (Abb 1). Danach wurden fünf Milben auf die Bienen aufgesetzt und die Zeit bestimmt, bis die Parasiten von ihrem Wirt abfielen. Als Referenz diente die Abfallzeit von unbehandelten Kontrollbienen. Bei einer Behandlung mit Fluvalinat unterschieden sich die Abfallzeiten und lagen je nach verwendeter Varroalinie zwischen 6 und 1218 Minuten. Die längsten Überlebenszeiten wurden bei Milben aus Bienenständen gefunden, in denen die Behandlung mit Apistan unwirksam geblieben war (Tabelle I). Bei einer Behandlung mit Acrinathrin wurden ebenfalls signifikante Unterschiede gefunden, die mit dem Ausmaß der Resistenz gegenüber Fluvalinat zusammenfielen. Gegenüber Amitraz wurden keine Unterschiede gefun- 
den. Diese einfache Methode des "Vergleichs der mittleren Überlebensdauer" kann auch auf andere Akarizide angewandt werden. Hiermit können der Zeitverlauf der Resistenzentwicklung verfolgt und die Imker besser informiert werden.

\section{Apis mellifera / Varroatose / Resistenz}

\section{RÉFÉRENCES}

Abed T, Ducos de Lahitte J (1993) Détermination de la $\mathrm{DL}_{50}$ de l'amitraz et du coumaphos sur Varroa jacobsoni Oud au moyen des acaricides Anti-varroa (Schering) et Perizin (Bayer). Apidologie 24, 121-128

Bassand D (1993) Del buen o mal uso del fluvalinato contra Varroa: estudio de los riesgos de aparicion de resistencia. Vida Apic 61, 18-22

Borneck R, Merle B (1990) Essais sur Apistan en 1988. Apiacta 25, 16-25

Colin ME (1983) Étude du premier foyer français de varroatose de l'abeille. Bull Acad Vét 56, 89-93

Faucon JP (1995) Communication Congrès FNOSAD Saverne 3 mars 1995. Étude de la baisse de l'efficacité de l'Apistan en France dans la région frontalière avec l'Italie.

Faucon JP, Drajnudel P, Fléché C (1995) Mise en évidence d'une diminution de l'efficacité de I'Apistan utilisé contre la varroose de l'abeille (Apis mellifera) Apidologie 26, 291-296

Faucon JP, Fléché C (1988) L'amitraz dans le traitement de la varroase de l'abeille. Rev Méd Vét 139, 389-406

Fluri P (1995) Tessin : efficacité de l'Apistan et du Bayvarol en régression. J Suisse Apic 6, 198-199

Lodesani M, Colombo M, Spreafico M (1995) Ineffectiveness of Apistan ${ }^{\circledR}$ treatment against the mite Varroa jacobsoni Oud in several districts of Lombardy (Italy). Apidologie 26, 67-72

Milani N (1995) The resistance of Varroa jacobsoni Oud to pyrethroids: a laboratory assay. Apidologie 26, 415-429

Nelson DL, Jay SC (1989) The effect of colony relocation on loss and disorientation of honeybees. Apidologie 20, 245-250

Peng YS, Fang $Y, X u S, G e L$ (1987) The resistance mechanism of the Asian honeybee, Apis cerana Fabr, to an ectoparasitic mite, Varroa jacobsoni Oudemans. J Invertebr Patho/ 49, 54-60

Richez P, Le Conte Y, Colin B (1995) Efficacite thérapeutique d'une nouvelie formulation (Apivar) destinée au traitement de la varroose de l'abeille. Santé Abeille $149,210-212$

Sakofski F, Koeniger N, Fuchs S (1990) Seasonality of honeybee colony invasion by Varroa jacobsoni Oudemans. Apidologie 21, 547-550

Vandame R, Colin ME, Belzunces LP, Jourdan P (1995) Mise au point d'une méthode pour évaluer la sensibilité de Varroa jacobsoni aux acaricides : application au fluvalinate. Carnets CAR/46,5-11 